\title{
PHOTOBIOMODULATION PROTECTS THE RETINA FROM LIGHT-INDUCED PHOTORECEPTOR DEGENERATION
}

\author{
Rizalyn Albarracin ${ }^{1,2}$ Janis Eells $^{4}$ and Krisztina Valter ${ }^{1,2,3}$ \\ From the ${ }^{1}$ Research School of Biology, ${ }^{2}$ ARC Centre of Excellence in Vision Science, \\ ${ }^{3}$ Medical School, The Australian National University, Canberra, Australia, ${ }^{4}$ Department of \\ Biomedical Sciences University of Wisconsin, Milwaukee, USA
}

\author{
Corresponding Author: \\ Krisztina Valter \\ Division of Biomedical Sciences \& Biochemistry \\ Research School of Biology \\ The Australian National University \\ GPO Box 475 \\ Canberra ACT 2601 \\ Australia \\ valter@rsbs.anu.edu.au
}

Word Count: 9875

Grant Support: This research was supported by the Australian Research Council through the ARC Centre of Excellence in Vision Science (CE0561903) and the Foundation for Fighting Blindness (FFB TA-NP-1107-0435-UWI). 


\section{ABSTRACT}

PURPOSE. We tested the hypothesis that near-infrared (NIR) light treatment (photobiomodulation) would attenuate bright light damage in the albino rat retina. METHODS. Young adult Sprague-Dawley (SD) albino rats were raised in dim (5Ix) cyclic light then exposed to bright (1000lx) continuous light for $24 \mathrm{~h}$. Animals were treated with $670 \mathrm{~nm}$ light $\left(9 \mathrm{~J} / \mathrm{cm}^{2}\right)$ using an LED array before, during or after light exposure. Retinas were examined for function, structural changes, cell loss, and markers of stress and inflammation at 1 week and 1 month after exposure to damaging white light. RESULTS. Bright light caused photoreceptor-specific cell death in control retinas. Significant upregulation of stress and neuroprotective factors, and the presence of activated microglia was also noted following light damage. Photobiomodulation profoundly attenuated histopathological alterations in all three treatment groups. NIR treatment also abolished microglial invasion of the retina and significantly reduced the presence of stress and neuroprotectant molecules. Bright light-induced reductions in photoreceptor function were significantly ameliorated by photobiomodulation in animals treated before and during light damage. Photoreceptor function was initially reduced in animals treated following bright light damage but recovered by one-month post exposure. CONCLUSIONS. NIR photobiomodulation is protective against bright light-induced retinal degeneration even when NIR treatment is applied after light exposure. This protective effect appears to involve a reduction of cell death and inflammation. Photobiomodulation has the potential to become an important treatment modality to prevent or treat light-induced stress in the retina. More generally, it could be beneficial in the prevention and treatment of retinal conditions involving inflammatory mechanisms. 


\section{INTRODUCTION}

It has been known since the 1960's that exposure to excessive light causes photoreceptor damage and death ${ }^{1,2}$. The severity of damage depends on multiple factors, including the intensity or the spectral distribution of the light, the duration of exposure, the retina's history of previous light experience ${ }^{3,4}$. Lesions produced by light damage (LD) are characterized by the damage or death of photoreceptors, the disorganisation or loss of RPE cells, Muller cell gliosis, the disruption of the outer limiting membrane (OLM), and the obstruction of the underlying choroidal vessels. These structural changes are accompanied by the accumulation of microglia and lymphocytes in the retinal and choroidal vessels, typically in areas with the most severe damage. Once the blood-retina barrier (BRB) is disrupted, the invasion of the outer retina by activated microglia becomes apparent ${ }^{5-9}$.

Photobiomodulation is the process, by which specific wavelengths of light are absorbed by cellular photo-acceptor molecules resulting in the activation of signalling pathways culminating in biological changes in the cell. A typical example would be the absorption of light by chlorophyll in plants, initiating photosynthesis. In animals, light absorbed by opsins, initiates phototransduction in photoreceptors. Apart from these specialised photoreceptor molecules, there are more than 50 non-specialised photoacceptor molecules in mammalian cells. Water, haemoglobin, oxyhaemoglobin and melanin all absorb light at a wide range of wavelengths. Between 600-1000nm wavelengths however, their absorption curve is at the minimum, providing a 'window' for other photosensitive molecules to be activated by light ${ }^{10}$. Cytochrome $\mathrm{c}$ oxidase, the terminal electron-acceptor in the mitochondrial electron transport chain, is a key photoacceptor molecule which has been shown to be activated by far-red to NIR light leading to biological changes ${ }^{11,12}$. 
The cellular responses to light in the range of far-red to near-infrared photon therapy include an increase in metabolic rate, cell migration and proliferation and the production and secretion of proteins. Zhang and colleagues have demonstrated changes in gene expression profiles in human fibroblasts following exposure to $628 \mathrm{~nm}$ light for 3 days, describing up-regulation in mitochondrial respiratory chain and antioxidant genes, and down-regulation in some apoptosis and stress response genes ${ }^{13}$. Among the proteins, some neuro-protective factors, including basic fibroblast growth factor (FGF2) and nerve growth factor (NGF) have been shown to be up-regulated ${ }^{14}$. The use of $670 \mathrm{~nm}$ light has also been demonstrated to protect neuronal cells after cyanide or TTX poisoning ${ }^{12}$.

In vivo experiments showed improved quality of wound healing in rat skin when treated with low-energy ruby laser ${ }^{15}$ or with Gallium laser at 685 or $830 \mathrm{~nm} \mathrm{light}^{16}$. Following spinal cord injury, the use of $810 \mathrm{~nm}$ light resulted in increased regeneration and functional recovery in the rat ${ }^{17}$. Eells and colleagues showed that $670 \mathrm{~nm}$ LED light was protective to photoreceptors in the methanol-induced degeneration rat model ${ }^{18}$, others demonstrated that NIR light promoted healing after laser lesions in primate retina ${ }^{19}$.

Clinically, the use of NIR light has been shown to be beneficial in the treatment of gingival incisions ${ }^{20}$, post-transplantational oral mucositis ${ }^{21}$, radiation ulcers of the $\operatorname{skin}^{22}$, acne vulgaris dermatitis ${ }^{23}$. It promoted wound healing ${ }^{24}$, peripheral nerve repair following trauma $^{25-27}$ and in carpal tunnel syndrome ${ }^{28,29}$. NIR treatment is also used in sport medicine and rehabilitation to treat acute soft tissue injuries ${ }^{30}$. It is worth noting that the presence of inflammation is common to these conditions.

Inflammatory events are present in the light-stressed retina ${ }^{5,6,9}$. Moreover, antiinflammatory measures such as the administration of microglial inhibitor Naloxone, have been shown to reduce light-induced damage in the retina ${ }^{31,32}$, indicating that inflammation is a critical factor in this model. The purpose of this investigation was to test the hypothesis 
that $670 \mathrm{~nm}$ photobiomodulation will ameliorate light-induced retinal damage and modulate cellular immune response in the rat retina.

\section{MATERIALS AND METHODS}

\section{Animals}

All procedures were in accordance with the ARVO Statement for the Use of Animals in Ophthalmic and Vision Research, and with the requirements of The Australian National University Animal Experimentation Ethics Committee. Albino Sprague-Dawley (SD) rats were born and raised in low (5lx) light levels with a $12 \mathrm{~h}$ light, $12 \mathrm{~h}$ dark cycle. Food and water were available ad libitum. Once animals reached P100-P120, they were divided into 6 groups. Three groups were designed to serve as baseline comparisons. In the first group animals were not exposed to either NIR or damaging white light (CONTROL group, $n=16$ ). In the second group, animals were exposed to damaging white light only (LD group, $n=16$ ) and in the third group they were exposed to NIR light only (NIR CONTROL group, $n=16$ ). In the remaining groups animals were exposed to damaging bright white light $(\mathrm{BL})$ as well as treated with NIR following one of three paradigms described below.

\section{Light Damage}

Animals $(n=19)$ were transferred to individual transparent cages with food placed on the cage floor and water was provided in transparent bottles to avoid shading of the light entering the cage. Fluorescent light tubes (18W, Cool White) were placed $200 \mathrm{~mm}$ above the bottom of the cage, so that the light intensity reached 1000 lx at the cage floor. Prior to light exposure animals were dark adapted overnight. Light exposure started at 9:00AM in all experimental paradigms. Animals were exposed to bright light for 24 hours and then returned to low light level environment (5lux) to recover for 1 week or 1 month. 


\section{NIR Treatment Paradigms}

Animals were wrapped in a cloth to aid manual handling, and placed under the 670nm LED array (Quantum Devices, WI, USA). Animals were positioned so that their eye level was approximately $2.5 \mathrm{~cm}$ away from the light source and were exposed to the light for 3 minutes at $60 \mathrm{~mW} / \mathrm{cm}^{2}$. This treatment protocol produced an energy fluence of $9 \mathrm{~J} / \mathrm{cm}^{2}$ at eye level. The first group of animals were treated with NIR $1 x$ daily on 5 consecutive days prior to $\mathrm{BL}$ (Pre-conditioned group, $\mathrm{n}=16$ ). The second group was treated immediately after the cessation of $\mathrm{BL}$ once a day for 5 days (Post-conditioned group, $\mathrm{n}=16$ ). The third group was treated 1 day prior to $\mathrm{BL}$, then $2 \mathrm{x}$ daily during and immediately after BL (Mid-conditioned group, $\mathrm{n}=16$ ). In the NIR Control group animals were treated with NIR light once daily for 5 days but were not exposed to BL (NIR Control group, $n=16$ ). Some animals $(n=3)$ were sham-treated prior to exposure to bright light. They were wrapped in towel and kept under an unswitched LED array box for 3 minutes.

\section{Electroretinography}

The function of photoreceptors was assessed by full-field flash-evoked electroretinogram (ERG). Animals were dark-adapted overnight and prepared for recording in dim red illumination as described previously ${ }^{33-35}$.

A series of single flashes of sufficient intensity to elicit saturated a-wave and b-wave responses $\left(44.5 \mathrm{cds} / \mathrm{m}^{2}\right)$ was used to record 'mixed' responses to assess both rod and cone photoreceptor function. As previously reported, the a-wave represents rod function in these 'mixed' responses ${ }^{36,37}$. All animals were recorded prior to the commencement of experimental procedures (Baseline recording) and then 1 week ( $n=8 /$ group) or 1 month ( $n=8 /$ group) after $B L$ exposure, regardless of NIR treatment paradigm. 


\section{Tissue Collection}

Tissue was collected 1 week or 1 month following BL exposure for histological and immunohistochemical evaluation. Animals were euthanized with an overdose of sodium pentobarbital (>60 mg/kg, intraperitoneal). Eyes were marked at the superior aspect of the limbus for orientation, enucleated and immersion-fixed in $4 \%$ paraformaldehyde in $0.1 \mathrm{M}$ phosphate-buffered saline (PBS) at $\mathrm{pH} 7.4$ for $2 \mathrm{~h}$.

To prepare for cryosectioning, eyes were rinsed thrice in $0.1 \mathrm{M}$ PBS and left in a $15 \%$ sucrose solution overnight for cryoprotection. The next day, eyes were embedded in Tissue-Tek OCT Compound (Sakura Finetek, Tokyo, Japan), and snap frozen in liquid nitrogen. They were cryosectioned in the sagittal plane, to allow a dorsal to ventral observation of the retina at $16 \mu \mathrm{m}$ or $60 \mu \mathrm{m}$ using Leica CM1850 cryostat (Leica Microsystems, Nossloch, Germany). Sections were mounted on gelatin and poly-L-lysinecoated slides and dried overnight at $50^{\circ} \mathrm{C}$ before being stored at $-20^{\circ} \mathrm{C}$.

\section{Histology and Structural Measurements}

\section{Toluidine Blue Staining}

To evaluate structural changes in the retina, Toluidine Blue staining was performed. Sections were immersed in $70 \%$ ethanol for 5 min for dehydration. The slides were then washed with $\mathrm{H}_{2} \mathrm{O}$ for 5 min before 2 drops of $1 \%$ Toluidine Blue were added and left to stain the sections for $5 \mathrm{~min}$. Once the sections had taken a purple blue colour, the sections were immediately washed with $\mathrm{H}_{2} \mathrm{O}$. The stained sections were cover slipped in glycerolgelatin medium (Sigma Chemical Co., St. Louis, MO, USA).

\section{Retinal Thickness Measurements}

To assess retinal thickness, cryosections were labelled with the DNA-specific dye bisbenzamide (Hoechst). Sections were thawed at room temperature before washing in 
$70 \%$ ethanol for 15 min, followed by a 5 min wash in distilled $\mathrm{H}_{2} \mathrm{O}$ and two 5 min washes in 0.1 M PBS. The sections were then incubated for 2 min with bisbenzamide (1:10 000), washed in 0.1 M PBS, and cover slipped with a glycerol/gelatin medium. Retinal thickness measurements were made on digital images of stained cryosections. At each location, we recorded the thickness of the outer nuclear layer $(\mathrm{ONL})$, as well as the thickness of the retina, from inner to outer limiting membrane (ILM-OLM). The ratio of ONL and ILM-OLM was used for analysis to account for obliquely-cut sections. In at least 4 sections per animal, we took 10 measurements from each section, approximately $100 \mu \mathrm{m}$ apart along the retina, (a total of 40 measurements per animal). Results from 8 animals from each group were averaged and analysed by the statistical method described below.

\section{OS lengths measurement}

Quantitative analysis of the length of the OS (outer segments) was performed in tissues cryosectioned at $60 \mu \mathrm{m}$ labelled with Toluidine Blue. Sections were examined and measured using LM-Zeiss Axioskop (Jena, Germany) with Spot CCD camera (MI, USA) with a calibrated 40X objective. Images were taken from both superior and inferior retina $(n=4)$. Spot CCD software was used to trace the length of the OS with a mean 3 equispaced measurements taken from each area. The average OS length from 4 animals was used for statistical analysis.

\section{Immunohistochemistry of Retinal Sections}

Cryosections were labelled with antibodies against glial fibrillary acidic protein (GFAP, rabbit polyclonal) (1:700, DAKO, CA, USA), basic fibroblast growth factor (FGF-2, mouse monoclonal) (1:200, Upstate Biotechnology, USA), ciliary neurotrophic factor (CNTF, goat polyclonal) (1:200, R\&D Systems, MN, USA), ionized calcium binding adaptor

molecule 1, (IBA1, mouse monoclonal) (1:500, Abcam, MA, USA) and CD68, also known 
as ED-1, (mouse anti-rat monoclonal, 1:200, Millipore, CA, USA) using a protocol previously described ${ }^{35}$. Briefly, sections were blocked with $10 \%$ normal goat serum for $1 \mathrm{~h}$ before being incubated with primary antibody for $24 \mathrm{~h}$ at $4^{\circ} \mathrm{C}$. Sections were treated with an antibody to either rabbit IgG conjugated with Alexa Fluor 594 or mouse IgG conjugated with Alexa Fluor 488 (1:1000, Molecular Probes, OR, USA) for $24 \mathrm{~h}$ at $4^{\circ} \mathrm{C}$ before incubation with the DNA-specific dye bisbenzamide (1:10 000) for 2 min.

\section{RNA Isolation and cDNA Synthesis}

Retinas were collected and stored in RNAlater ${ }^{\circledR}$ (Ambion, Applied Biosystems, Foster City, CA) overnight at $4^{\circ} \mathrm{C}$. RNA isolation was then performed using TRIzol Reagent (Invitrogen, Carlsbad, CA). The retinas were homogenised in $1.5 \mathrm{ml}$ tube with $200 \mu \mathrm{l}$ of Trizol on ice, followed by further addition of $660 \mu$ l Trizol and $160 \mu$ l chloroform. The tube was vortexed for $20 \mathrm{~s}$, allowed to stand for 7 minutes at room temperature and then centrifuged at $13,000 \mathrm{x} \mathrm{g}$ for 10 minutes at $4^{\circ} \mathrm{C}$. The resulting supernatant was subsequently removed and transferred into a clean $1.5 \mathrm{ml}$ tube with half of its volume of $100 \%$ absolute ethanol. The tube with its content was vortexed briefly prior to performing purification and DNAse treatment steps, as outlined in the RNAqueous-micro kit protocol (Ambion, Applied Biosystems Foster City, CA). The purified and DNAse-treated RNA was quantified on a spectrophotometer (ND-1000; Nanodrop Technologies, Wilmington, DE) followed by analysis of its integrity using a bioanalyser (2100 Bioanalyzer; Agilent Technologies, Santa Clara, CA). The synthesis of cDNA was achieved by reverse transcribing $1 \mu \mathrm{g}$ of total RNA using the Superscript ${ }^{\circledR}$ III Reverse Transcriptase First Strand Synthesis kit following the prescribed manufacturer's protocol (Invitrogen Life Technologies, Carlsbad, CA). 


\section{Real Time Quantitative Polymerase Chain Reaction (RT-qPCR)}

Validation of the changes in expression of stress-related Gfap and protective Fgf-2 genes were determined by RT-qPCR using Taqman ${ }^{\circledR}$ probe IDs Rn00566603_m1 for Gfap and Rn00570809_m1 for Fgf-2, combined with the Gene Expression Master-Mix (Applied Biosystems, Foster City, CA) with the use of StepOne Plus qPCR machine with the StepOne software v2.1 (Applied Biosystems). Glyceraldehyde 3-phosphate dehydrogenase (Gapdh) was used as a reference gene. To account for variability, Taqman amplification assay was performed in duplicates (individual sample variability) with triplicate biological samples to account for individual animal differences. The fold changes were determined using comparative cycle threshold (Ct; delta-delta ct).

\section{Microscopy}

Images of the immuno-labelled retinal sections were obtained using Carl Zeiss confocal microscope (Germany) and LM Zeiss Apotome (Germany). Only samples that were processed and imaged concurrently were used for analysis. During image collection, the photomultiplier settings were kept constant to allow more accurate comparison of protein levels.

\section{Statistical Analyses}

Data were analyzed using a two-tailed Student's t-test with $P<0.05$ considered to represent a statistically significant difference. All data are presented as the mean \pm 1 SEM. 


\section{RESULTS}

\section{NIR photobiomodulation preserved retinal structure in all 3 treatment paradigms}

Figure 1A depicts retinas of animals not exposed to BL nor treated with NIR (control), animals exposed to BL (LD), animals not exposed to BL, but treated with NIR (NIR treated), and animals exposed to BL and treated with NIR following one of the three treatment paradigms (Precon, Midcon, Postcon). Histological labelling of cryosections with Toluidine blue showed that exposure to BL led to structural damage in the outer retina (Fig 1A, B). The severity of the damage was uneven along the retina. One week after light exposure, an area of high-density cell death was detectable 1-2mm supero-temporal to the optic disc (depicted in Figure 1A, C) that exhibited significant thinning of the ONL in BLexposed, non-treated retinas. In this area both inner (IS) and outer segments (OS) were lost and only a few rows of photoreceptors remained. The retina was severely distorted, the outer limiting membrane and the retinal pigment epithelial cell row were disrupted and the BRB appeared to be compromised (Fig 1A \& B 'LD'). Similar pattern and severity of structural damage was observed in LD-exposed retinas with sham NIR treatment. Quantitative analyses indicate that there were no significant differences in the OS lengths and ONL thickness between the non-treated and sham NIR-treated retinas with LD exposure. In areas, outside this "hot spot", the retinal changes were minor, limited to the shortening and distortion of the photoreceptor outer segments (not shown). These images demonstrate that NIR treatment alone did not cause any structural alterations in the retina. In light-exposed retinas treated with NIR, the preservation of the ONL was evident, whereas the IS/OS were only slightly shortened and mildly disrupted in all three treatment paradigms. 
The cumulative effect of photoreceptor cell death was assessed by the measurement of the outer nuclear layer (ONL) thickness (Fig 1C). Toluidine blue staining was used to detect surviving cells 1 week after BL exposure (Fig 1A). The panel depicting a BL exposed non-treated retina shows a severe disruption and reduction of the photoreceptor population in the hot spot area (Fig 1A, B 'LD'). NIR treatment of BL-exposed retinas profoundly attenuated photoreceptor cell loss in all treatment paradigms. Figure $1 \mathrm{C}$ shows the average ONL thickness along the retina in 8 animals in each treatment group. BL exposed, non-treated retinas showed a significant thinning of the ONL in all areas of the retina; in the inferior retina, there was a $25 \%$ loss, while in the superior retina there was a $50 \%$ reduction of thickness, and in the hot spot area ONL thickness was reduced by $75 \%$ compared to controls. NIR-treated BL exposed animals exhibited a small decrease in ONL thickness. However, ONL thickness in NIR-treated BL-exposed animals was not statistically different from values measured in Control animals.

Figure 1D shows the average OS length values along the retina for 8 animals in each group 1 week after BL exposure. In BL exposed animals, OS length was close to $60 \%$ shorter in the inferior retina when compared to corresponding areas of control retinas, and $70-75 \%$ shorter in the penumbra of the hot spot in the superior retina compared to controls, with a complete loss of the IS/OS in the hot spot area. NIR treatment ameliorated OS damage in BL exposed animals. In the pre-conditioned group OS lengths were not significantly different from control animals not exposed to BL. Retinas in the midconditioned group exhibited an approximately $25 \%$ decrease in OS length compared to control. In the post-conditioned group, OS length was reduced to $50 \%$ of non-BL control values. In the hotspot region, the length of OS dropped by around $60 \%$, however there was no complete loss of OS at any of the retinas in this group. 


\section{NIR photobiomodulation attenuated LD-induced retinal functional loss}

Figure 2 summarises the changes in photoreceptor function 1 week after exposure to BL. Panel A shows typical recordings of selected animals from each group. Dotted lines show baseline recording and solid lines show post-BL recordings of the same animals. Grey lines represent non-treated BL-exposed animals, black lines represent NIR treated lightstressed retinas 1 week post-exposure. The figure also demonstrates that there was a significant loss of retinal function following $\mathrm{BL}$ exposure in preconditioned and midconditioned animals, however, the NIR treated animal exhibited a more moderate loss compared to the non-treated animal, showing significantly better photoreceptor function when compared to LD group. In the post-conditioned group, at 1 week after BL, photoreceptor function of NIR treated animals was indistinguishable from that of nontreated LD animals.

Figure $2 \mathrm{~B}$ shows the average a-wave amplitudes assessed by the dark-adapted full-field flash ERG in 8 animals in all experimental groups. Changes were expressed as the ratio of amplitudes measured 1 week after BL exposure and amplitudes measured pre-exposure on the same animal, thus a value of 1 represent no change. NIR treatment alone did not have a significant effect on photoreceptor function. Retinas, exposed to BL but not treated with NIR showed a significant loss of photoreceptor function, decreasing to less than $20 \%$ of baseline value 1 week after BL exposure. In the NIR treated groups the effect of BL on photoreceptor function varied. In the pre-conditioned group, the amplitude of a-wave decreased to $56 \%$ of baseline values 1 week after BL. Although the decrease from baseline was significant, the post LD values of the a-waves in the NIR pre-conditioned group were significantly greater than that measured in the LD group. In the NIR midconditioned group, photoreceptor function was reduced to $60 \%$ of baseline values indicative of photoreceptor protection compared to the LD group. The NIR post- 
conditioned group exhibited significant photoreceptor dysfunction relative to baseline recordings. In this group, there was a $70 \%$ reduction in the a-wave amplitude at 7 days post LD. The relative a-wave amplitudes of the NIR post-conditioned group were not significantly different from those measured in the non-treated BL group, thus NIR postconditioning did not offer functional protection for photoreceptors at this early stage.

The effects of NIR treatment on the functional response downstream from the photoreceptors was also assessed by measuring the b-wave amplitudes in NIR-treated retinas $7 d$ after LD exposure. The relative b-wave amplitude was analysed and presented in the same manner as the relative a-wave values were assessed (please refer to previous section). As shown in Figure 2C, b-wave response in the non-treated LD retinas was reduced by $73 \%$ from the baseline value. There was a slight variation observed in responses in b-wave amplitudes from the retinas in NIR-treated groups. The reduction of b-wave response in the Midconditioned retina was only $20 \%$ while the Precon group showed $30 \%$ loss of b-wave amplitude. Conversely, the Postcon retinas showed the most severe reduction, b-wave response reaching $30 \%$ of control values, a level similar to the non-treated LD retina. There was no change observed in the NIR-treated only control.

\section{NIR photobiomodulation mitigated retinal stress and modulated}

\section{neuroprotectant regulation}

We observed the levels of two known retino-protectant proteins, ciliary neurotrophic factor (CNTF) and basic fibroblast growth factor (FGF-2) and the glial fibrillary acidic protein (GFAP), a known stress marker, following light exposure and assessed the effects of NIR on these protein levels. Ciliary neurotrophic factor was not detectable in the normal retina. Treatment with NIR alone did not change the pattern of protein expression and did not increase its concentration. Following light exposure, there was an up-regulation of protein levels in astrocytes, and Muller cells, exhibiting a strong, punctate labelling along 
the Muller cell processes. In the penumbra of the hot spot, CNTF labelling in the retinal tissue was limited to the OS's of photoreceptors. In animals exposed to BL and treated with NIR, CNTF protein up-regulation varied. The least up-regulation was found in the preconditioned group where CNTF was barely detectable in the retinal tissue. Retinas of the mid-conditioned group showed stronger presence of the protein, localised in the OS's. Retinas of the postconditioned group demonstrated the strongest up-regulation in the photoreceptor OS layer and the astrocytes, where the labelling showed similar pattern and intensity found in the penumbra region of the control retina (Fig 3D).

GFAP labelling was limited to astrocytes, in the innermost layer of the retina in healthy retinas. In control animals and in animals treated with NIR alone, GFAP labelling showed normal pattern as demonstrated in Fig $3 \mathrm{C}$ in red. In light-stressed retinas, labelling of Muller cell processes became apparent. In non-treated, BL-exposed retinas a strong upregulation of GFAP was detectable in astrocytes and in Muller cells 1 week after light exposure. Labelling was most prominent in the hot spot, where the entire Muller cell became GFAP+ from the inner limiting membrane to the outer retina and the subretinal space, where activated Muller cells started to form glial scar (Fig 3C, red). In regions outside the hot spot, labelling was present in the Muller cells but was more muted (not shown).

In the NIR treated animals, in all three treatment paradigms, GFAP labelling was upregulated and was present in the Muller cells, but localised to the inner layers of the retina predominantly, not reaching the outer processes of Muller cells. However, there was a noticeable gradient between treatment paradigms, with a more moderate change in the pre-conditioned and mid-conditioned animals, and a more significant up-regulation in the post-conditioned animals, though even here, the levels of GFAP regulation did not reach the levels found in non-treated BL-exposed animals. 
FGF-2 is normally present in retinal macroglia (Muller cells and astrocytes), ganglion cells and RPE, but not in photoreceptors ${ }^{38-40}$. In control retinas, FGF-2 labelling was prominent in the Muller cell bodies, in the INL (Fig 3C, green). Following exposure to damaging light, FGF-2 protein was up-regulated in the ONL. This up-regulation was noted along the retina, but was most prominent in the hot spot.

NIR treatment alone did not cause a change in FGF-2 protein expression. In animals from the pre-conditioned group, FGF-2 up-regulation was not detected in the ONL. In the mid-conditioned group only a moderate increase in FGF-2 labelling was detectable in the INL and ONL of the hot spot region, but not outside this area. There was a significant upregulation of FGF-2 protein in the ONL. In the post-conditioned group, similar to the one seen in control light-exposed retinas.

To validate the expression of the FGF-2 and GFAP proteins described in the previous sections, the regulatory effects of NIR treatment in Gfap and Fgf-2 genes were assessed quantitatively 1 week post BL exposure using RT-qPCR. We observed an increase in gene expression of both Fgf-2 and Gfap of both light exposure and NIR treated retinae. Figure $3 \mathrm{~A}$ shows that in the non-treated LD retina, there was a 5.8 -fold increase in the expression of Fgf-2 gene. However, the NIR-treated groups exhibited varied levels of upregulation. There was a 3-fold increase in the Precon, 6.2-fold in Midcon and Postcon groups generated an 8.7-fold upregulation. When compared to the non-treated LD retina, Precon group showed a significant difference. There was no change observed in the level of expression of Fgf-2 gene in the NIR-treated control. Similar pattern of gene expression was observed in the stress-related Gfap gene following LD exposure and NIR treatment (Figure 3B). An increase of 12.2-fold was seen in the non-treated, light-stressed retina (LD) while the upregulation in Precon was slightly down to 8.9-fold, and a more modest 
increase of 12-fold in the Midcon-treated retinas and 21.8-fold in the Postcon group (Figure 3B). No fold change was observed in the NIR-treated control group.

\section{NIR light treatment mitigated light-induced inflammatory reaction}

Antibodies against ED-1, macrophage and monocyte marker and IBA1, a microglia marker were used to assess the presence of inflammatory cells in the light-stressed retina (Figure 4). In control non-BL exposed non-treated animals, very few monocytes were seen and those present were limited to the choroidal vessels. In BL-exposed retinas, monocytes were detectable in both the choroidal and retinal vessels in the area of the incipient hot spot immediately after BL exposure. By 1 week post-exposure, a breakdown of the bloodretina barrier was apparent in the hot spot area, where monocytes invaded the retinal tissue and were present in the severely damaged ONL (Figure 4A). NIR treatment alone did not change monocyte numbers in the choroidal, retinal vessels or in retinal tissue. In BL-exposed, NIR-treated animals, there was a slight increase in monocyte numbers both in retinal and choroidal vasculature, however this increase was significantly less than the level seen in light-exposed control animals. In addition, retinal invasion of macrophages/monocytes was not detectable in any of the treated animals (Figure 4A). Quantitative assessment of monocyte population was performed by counting ED-1+ profiles in different layers of the retina and in the choroid of 4 animals in each experimental group and summarised in the graph (Figure 4B). Light exposure caused a significant increase in the presence of monocytes in the choroid and inner retina as well as in the severely damaged ONL of the hot spot. Although, there was a slight increase in the number of monocytes both in the choroidal and retinal vessels in the NIR treated lightstressed retinas, this increase was significantly less than in non-treated, BL exposed retinas. Moreover, there were no ED-1+ profiles present in the retinal tissue in any of the NIR-treated retinas. 
To establish the source of the activated microglia present in the retina, IBA1 labelling was performed (Figure 4C). This antibody can detect a protein specifically expressed in microglia and macrophages and to discriminate resting and activated microglia. Figure $4 \mathrm{C}$ shows the distribution of microglia in the retina. In control retinas, microglia present in choroidal and retinal vessels exhibited a ramified appearance, typical to resting microglial cells. After exposure to BL, the majority of microglial cells that were detected in the retina showed amoeboid configuration, typical to cells in an activated phase. When we quantified resting and activated microglia in the retina, as shown in Figure 4D, we found that NIR treatment alone did not change the numbers or the activity status of microglia when compared to control. Following exposure to $\mathrm{BL}$, the number of microglia did not increase significantly, however, the ratio of activated cells increased significantly, reaching $85 \%$ of all microglia present. In the preconditioned and midconditioned groups the ratio of activated cells increased significantly, though it only reached 66 and $57 \%$ respectively, of the total microglial population detected. In the postconditioned group, the number of microglial cells and the ratio of activated cell within their population were similar to the nontreated BL-exposed group.

\section{Long-term effects of NIR treatment on the light-stressed retina}

To gauge the extent of the protective effect of NIR treatment, we assessed retinas 1 month after $\mathrm{BL}$ exposure. Figure $5 \mathrm{~A}$ summarises photoreceptor function in all 6 experimental groups, expressed as the average of ratio of post- and pre-exposure amplitude values for each animal, thus the value of 1 means no change. Again, NIR treatment alone showed no significant effect on photoreceptor function. In the light exposed but non-treated group, the average a-wave amplitude (top panel) fell to less than $15 \%$ of the pre-exposure value, which was not significantly different from values found at the 1 week timepoint. The preconditioned group showed a mild improvement from the 1 
week average value of $56 \%$ to $65 \%$ by 1 month postBL exposure. The midconditioned group showed no further improvement from the values measured at the 1 week postexposure time, it remained close to $60 \%$. However, when post-conditioned animals were allowed to recover for 1 month after BL exposure, there was a significant recovery in their photoreceptor function. While the average a-wave amplitude was $30 \%$ of baseline values at the 1 week time point, by 1 month postBL, the average value rose to $58 \%$ that was reaching the levels of the other 2 NIR treatment paradigm groups, becoming significantly greater than non-treated, BL damaged retinas.

The long term effect of NIR on the function of inner retinal cells was evaluated by measuring the b-wave amplitudes of NIR-treated animals 1 month after bright light exposure. As demonstrated in Figure 5A (bottom panel), the b-wave amplitude response from non-treated, LD-exposed animals showed further reduction from the values detected at 1 week postLD, reaching $10 \%$ of baseline values. Conversely, the b-wave responses in the NIR-treated groups showed no further loss of function. In the preconditioned and midconditioned groups, the values of the responses remained stable from the 1 week postLD levels. However, there was a significant improvement observed in the postconditioned group at 1 month after LD exposure. The b-wave amplitudes in this group reached $82 \%$ of baseline values, representing over $50 \%$ recovery from the 1 week postLD levels. Treatment with NIR alone did not cause any significant change in the photoreceptor function.

Figure 5B demonstrates retinal structure in the hot spot area, following 1 month of recovery. In the non-treated, light-exposed retina, the ONL was obliterated and RPE disrupted. RPE and ONL were both present in retinas treated with NIR, albeit the photoreceptor layer was thinner when it was compared to non-challenged control retinae. 
The outer segments showed organised appearance, compared to the disturbances observed in the midconditioned and postconditioned groups 1 week postBL.

Outer segment lengths measurements (Figure $5 \mathrm{C}$ ) showed that, there was a total loss of OS's in a wide area of the BL exposed retina, spanning over $5 \mathrm{~mm}$ length and centered by the original hot spot area. In the NIR-treated groups, the length of the OS's was reduced compared to control retinas, but was significantly longer compared to the non-treated light-exposed retina. Moreover, OS's were present along the retina in all three treatment groups.

To assess the cumulative effect of light and NIR treatment on the photoreceptor population, we measured the ONL thickness in all groups (Figure 5D). In the LD group, photoreceptor cells were lost in a large, $4 \mathrm{~mm}$ long area in the superior retina extending from the original hot spot area, whereas, there was a more moderate thinning of the ONL in the rest of the retina. The ONL thickness did not change greatly from the 1 week time point, in the NIR-treated retinas.

\section{DISCUSSION}

\section{Bright white light induced retinal damage}

Our findings in retinas exposed to bright continuous light for 24 hours are consistent with those reported in the literature. Three major events, characteristic of light-induced retinal damage are 1.) wide-spread death of photoreceptors, 2.) degeneration of pigment epithelial cells and 3.) reduction or total extinction of the ERG ${ }^{2,41}$. Rapp and Williams reported on the variable sensitivity of retinal regions to light ${ }^{42}$. Typically, the superior central portion of the retina shows a higher sensitivity than other areas ${ }^{9,42-44}$. This region has been shown to contain the highest concentration of ganglion cells ${ }^{45}$, and photoreceptors in this area possess longer outer segments ${ }^{46}$, which renders this part of the 
retina the functional area centralis in the rat. Recently we reported that in the light damage paradigm used in this study, the most severe, irreversible damage occurred in this central area of the retina. Moreover, severe, acute damage became the centre of a progressive degeneration ${ }^{9}$.

\section{$670 \mathrm{~nm}$ photobiomodulation induced retinoprotection}

This study demonstrated that $670 \mathrm{~nm}$ photobiomodulation ameliorates the damaging effects of bright continuous light on the retina. Treatment with $670 \mathrm{~nm}$ light before, during or even after exposure to $B \mathrm{~L}$ led to a significant reduction in photoreceptor cell death and prevented the severe disruption of the outer retina and the RPE. Photobiomodulation prevented the obliteration of the choroidal vascular network, thus assuring the maintenance of the blood-retina barrier. Photoreceptor structure was maintained in the treated groups, albeit the outer segments shortened, misaligned and some small vacuoles were present in the hot spot area. These changes however were reversible, because after a period of recovery in dim light, OS's became more organised and longer than at the early stages after light-stress. Our findings in the preconditioned group somewhat agreed with those reported by Qu et al ${ }^{47}$. Although they showed a reduced rate of photoreceptor loss and better retinal function in the NIR-treated animals in their light damage paradigm, they were not able to avoid the development of a hotspot that could be a source of long-term degeneration.

Photobiomodulation also reduced cell stress and inflammatory reaction in the retina. NIR treatment mitigated the up-regulation of GFAP in Muller cells, a known stress marker. It has also reduced the number of microglial cells in the retinal and choroidal vessels. Though it did not prevent the activation of microglia, it significantly reduced the number of activated cells and prevented their invasion into the outer retina. 
In conjunction with the maintenance of outer retinal structure, photoreceptor cell function was also preserved. In animals treated before (pre-conditioned) or during (midconditioned) BL, photoreceptor function was significantly better than non-treated controls at 1 week post-exposure and that function was maintained at the 1 month post-exposure time. In animals treated after BL (post-conditioned), photoreceptor function was significantly reduced 1 week post-exposure. However, by 1 month post-exposure, photoreceptor function recovered in this group as well, indicative of a protective effect similar to the other two treatment paradigms. In contrast, photoreceptor function remained diminished in the non-treated BL-exposed animals.

The apparent discrepancy between the state of retinal structure and its function in the postconditioned group prompted us to investigate possible mechanisms responsible for the reduction of photoreceptor function without apparent major structural damage. One such mechanism may possibly be through the up-regulation of neuroprotectants, such as ciliary neurotrophic factor (CNTF), basic fibroblast growth factor (FGF-2). Earlier studies have shown that both of these proteins are effective retinoprotectants ${ }^{48-51}$. It has also been demonstrated, that both of these factors have a direct effect on retinal function ${ }^{49,52-55}$. In our case, both CNTF and FGF-2 were up-regulated in the light-exposed non-treated group, similarly to earlier published data ${ }^{56}$. The up-regulation of both factors has been related to the reduction in retinal function ${ }^{52,53}$ and thus they could be factors in the functional changes observed in our animals. In the NIR treated animals, we were able to detect the up-regulation of CNTF in the photoreceptor OS layer and FGF-2 protein in the ONL at varying degree that could explain the difference in functional response in the three treatment groups. The most severe loss of function correlated with the highest level of CNTF and FGF-2 protein up-regulation. It has been shown that the neuroprotectant upregulation following an insult is only temporary ${ }^{52}$, and once the stress is removed, protein 
levels are gradually reduced. It is possible, that the transient loss of function in the postconditioned retinas are due, at least partly, to the increased presence of neuroprotectants in the outer retina, which allowed the survival and recovery of photoreceptors. Once the crisis was over, and their presence was no longer necessary, the level of these factors reduced, allowing the recovery of retinal function. In retinas exposed to damaging light, but not treated by $670 \mathrm{~nm}$ photobiomodulation, the white light induced damage of photoreceptors and RPE might possibly have overwhelmed the retinoprotective system, and despite the presence of these factors, a large number of photoreceptors were lost and thus retinal function could not recover.

\section{Potential Mechanism of Photobiomodulation}

Photobiomodulation, or low energy photon irradiation by far-red/near infrared (FR/NIR) light using low energy lasers or LED arrays, collectively termed "photobiomodulation", has been applied clinically in the treatment of soft tissue injuries and acceleration of wound healing for more than 30 years ${ }^{57}$. Recent studies demonstrated that low energy laser and LED NIR penetrates diseased tissues including the heart, spinal cord and brain ${ }^{58,59}$. NIR treatment has been documented to improve recovery from ischemic heart injury ${ }^{58}$, attenuate degeneration in the injured retina and optic nerve ${ }^{58,60}$ and improve recovery in experimental and clinical stroke ${ }^{59,61,62}$. Clinical trials in the treatment of radiation-induced mucositis and stroke have demonstrated therapeutic efficacy of $670 \mathrm{~nm}$ and $830 \mathrm{~nm}$ light administered at doses ranging from $3-6 \mathrm{~J} / \mathrm{cm}^{2}$. Thus, existing data demonstrate the therapeutic potential of NIR light.

The cytoprotective action spectrum of FR/NIR light corresponds with the cytochrome oxidase absorption spectrum ${ }^{57,63}$. Recent studies have shown that NIR irradiation produces redox alterations in the cytochrome oxidase molecule resulting in the activation of intracellular signaling cascades which culminate in improved mitochondrial function and 
increased synthesis of cytoprotective factors ${ }^{12,57,59,60,64-68}$. Others showed increased tissue concentrations of antioxidants (glutathione, mitochondrial superoxide dismutase [SOD2]) and cytoprotective growth factors ${ }^{69}$. Recently, we reported on gene regulatory effects of NIR treatment in the normal and light-stressed retina ${ }^{70}$. Our findings showed NIR had an effect on many intracellular pathways, among others a direct effect on antioxidant protection and the downregulation of a chemokine, $c c / 2$, that has been shown to induce leukocyte recruitment and activation ${ }^{71}$. Present study provided further evidence of the direct effect of NIR light on inflammation, by showing a reduction of monocyte recruitment and microglia activation in treated eyes.

Our earlier genechip study suggests, that NIR has no direct effect on the regulation of neuroprotectants, such as fgf-2 or cntf genes. This study confirmed the negative correlation between structural protection and FGF-2 regulation, that suggests that photobiomodulation does not act through neuroprotective pathways directly, but possibly at a point more upstream, thereby preventing, rather than mitigating cell damage.

More interestingly, our previous wide-scale gene analysis showed that NIR upregulated many noncoding RNAs (ncRNA) ${ }^{70}$. That could explain the lack of any apparent effect of the NIR treatment on the healthy, non-challenged retina. Further research is necessary to understand the exact role these 'non-coding' sequences may play in the retina, but one possible hypothesis presents itself, that while they don't have any apparent effect on healthy tissue, they may act as a pre-conditioning event that allows the modification of the regulation of potentially damaging genes in the presence of stress stimuli.

\section{Clinical implications}

The use of photobiomodulation remains controversial as a consequence of an incomplete understanding of its mechanisms of action, differences in treatment paradigms, 
wavelengths and mixed results in both the laboratory and clinic ${ }^{72}$. In addition, it is hard to assess the efficacy of the treatment in the clinic, due to the lack of appropriately controlled and blinded clinical trials. Photobiomodulation is used in many types of tissues targeting a variety of conditions using different wavelengths, dose and light sources (laser vs. noncoherent light), which makes standardisation near impossible. Despite the controversies, many successes have been achieved in a few areas clinically, specifically in three areas: 1) wound healing, 2) the relief of inflammation and 3) the reduction of neurogenic pain.

Present data suggest that treatment with $670 \mathrm{~nm}$ red light can lead to significant protection of the retina from light-induced damage. This treatment has the potential to reduce the adverse effects of bright light exposure moreover this non-invasive therapeutic modality has considerable promise for the treatment of retinal degenerative disorders and ocular inflammatory disease conditions. 


\section{REFERENCES}

1. Gorn RA, Kuwabara T. Retinal damage by visible light. A physiologic study. Arch Ophthalmol 1967;77:115-118.

2. Noell WK, Walker VS, Kang BS, Berman S. Retinal damage by light in rats. Invest Ophthalmol 1966;5:450-473.

3. Lanum J. The damaging effects of light on the retina. Empirical findings, theoretical and practical implications. Surv Ophthalmol 1978;22:221-249.

4. Reme CE, Weller M, Szczesny P, et al. Light-induced apoptosis in the rat retina in vivo. In: Anderson RE (ed), Degenerative Diseases of the Retina. New York: Plenum Press; 1995.

5. Ng TF, Streilein JW. Light-induced migration of retinal microglia into the subretinal space. Invest Ophthalmol Vis Sci 2001;42:3301-3310.

6. Gordon WC, Casey DM, Lukiw WJ, Bazan NG. DNA damage and repair in light-induced photoreceptor degeneration. Invest Ophthalmol Vis Sci 2002;43:3511-3521.

7. Langmann T. Microglia activation in retinal degeneration. J Leukoc Biol 2007;81:13451351.

8. Joly S, Francke M, Ulbricht E, et al. Cooperative phagocytes: resident microglia and bone marrow immigrants remove dead photoreceptors in retinal lesions. Am J Pathol 2009;174:23102323.

9. Rutar M, Provis J, Valter K. Brief exposure to damaging light causes focal recruitment of macrophages, and long-term destabilization of photoreceptors in the albino rat retina. Current Eye Research 2010;35:631-643.

10. Hamblin MR, Demidova TN. Mechanisms of low level light therapy. In: Hamblin MR, Waynant RW, Anders J (eds), SPIE; 2006:1-12.

11. Karu T. Primary and secondary mechanisms of action of visible to near-IR radiation on cells. J Photochem Photobiol B 1999;49:1-17.

12. Wong-Riley MT, Liang HL, Eells JT, et al. Photobiomodulation directly benefits primary neurons functionally inactivated by toxins: role of cytochrome c oxidase. $J$ Biol Chem 2005;280:4761-4771.

13. Zhang Y, Song S, Fong CC, Tsang CH, Yang Z, Yang M. cDNA microarray analysis of gene expression profiles in human fibroblast cells irradiated with red light. $J$ Invest Dermatol 2003;120:849-857.

14. Yu HS, Wu CS, Yu CL, Kao YH, Chiou MH. Helium-neon laser irradiation stimulates migration and proliferation in melanocytes and induces repigmentation in segmental-type vitiligo. $J$ Invest Dermatol 2003;120:56-64.

15. Mester E, Spiry T, Szende B, Tota JG. Effect of laser rays on wound healing. Am J Surg 1971;122:532-535. 
16. Viegas VN, Abreu ME, Viezzer C, et al. Effect of low-level laser therapy on inflammatory reactions during wound healing: comparison with meloxicam. Photomed Laser Surg 2007;25:467473.

17. Byrnes KR, Waynant RW, Ilev IK, et al. Light promotes regeneration and functional recovery and alters the immune response after spinal cord injury. Lasers Surg Med 2005;36:171185.

18. Eells JT, Henry MM, Summerfelt P, et al. Therapeutic photobiomodulation for methanolinduced retinal toxicity. Proc Natl Acad Sci U S A 2003;100:3439-3444.

19. Whelan HT. DARPA Soldier Self Care: Rapid Healing of Laser Eye Injuries with Light Emitting Diode Technology. RTO HFM Symposium on Combat Casualty Care in Ground Based Tactical Situations: Trauma Technologiy and Emergancy Medical Procedures. St Pete Beach, USA; 2004.

20. Neiburger EJ. Rapid healing of gingival incisions by the helium-neon diode laser. $J$ Mass Dent Soc 1999;48:8-13, 40.

21. Whelan HT, Connelly JF, Hodgson BD, et al. NASA light-emitting diodes for the prevention of oral mucositis in pediatric bone marrow transplant patients. J Clin Laser Med Surg 2002;20:319-324.

22. Schindl A, Schindl M, Pernerstorfer-Schon H, Mossbacher U, Schindl L. Low intensity laser irradiation in the treatment of recalcitrant radiation ulcers in patients with breast cancer--long-term results of 3 cases. Photodermatol Photoimmunol Photomed 2000;16:34-37.

23. Papageorgiou P, Katsambas A, Chu A. Phototherapy with blue (415 nm) and red (660 nm) light in the treatment of acne vulgaris. Br J Dermatol 2000;142:973-978.

24. Medrado AR, Pugliese LS, Reis SR, Andrade ZA. Influence of low level laser therapy on wound healing and its biological action upon myofibroblasts. Lasers Surg Med 2003;32:239-244.

25. Gigo-Benato D, Geuna S, Rochkind S. Phototherapy for enhancing peripheral nerve repair: a review of the literature. Muscle Nerve 2005;31:694-701.

26. Anders JJ, Geuna S, Rochkind S. Phototherapy promotes regeneration and functional recovery of injured peripheral nerve. Neurol Res 2004;26:233-239.

27. Anders JJ, Borke RC, Woolery SK, Van de Merwe WP. Low power laser irradiation alters the rate of regeneration of the rat facial nerve. Lasers Surg Med 1993;13:72-82.

28. Branco K, Naeser MA. Carpal tunnel syndrome: clinical outcome after low-level laser acupuncture, microamps transcutaneous electrical nerve stimulation, and other alternative therapies-an open protocol study. J Altern Complement Med 1999;5:5-26.

29. Irvine J, Chong SL, Amirjani N, Chan KM. Double-blind randomized controlled trial of low-level laser therapy in carpal tunnel syndrome. Muscle Nerve 2004;30:182-187. 
30. Simunovic Z, Ivankovich AD, Depolo A. Wound healing of animal and human body sport and traffic accident injuries using low-level laser therapy treatment: a randomized clinical study of seventy-four patients with control group. J Clin Laser Med Surg 2000;18:67-73.

31. Ni YQ, Xu GZ, Hu WZ, Shi L, Qin YW, Da CD. Neuroprotective effects of naloxone against light-induced photoreceptor degeneration through inhibiting retinal microglial activation. Invest Ophthalmol Vis Sci 2008;49:2589-2598.

32. Yang L, Kim JH, Kovacs KD, Arroyo JG, Chen DF. Minocycline inhibition of photoreceptor degeneration. Arch Ophthalmol 2009;127:1475-1480.

33. Walsh N, van Driel D, Lee D, Stone J. Multiple vulnerability of photoreceptors to mesopic ambient light in the P23H transgenic rat. Brain Res 2004;1013:194-203.

34. Jozwick C, Valter K, Stone J. Reversal of functional loss in the P23H-3 rat retina by management of ambient light. Exp Eye Res 2006;83:1074-1080.

35. Chrysostomou V, Stone J, Stowe S, Barnett NL, Valter K. The status of cones in the rhodopsin mutant $\mathrm{P} 23 \mathrm{H}-3$ retina: light-regulated damage and repair in parallel with rods. Invest Ophthalmol Vis Sci 2008;49:1116-1125.

36. Arden GB, Carter RM, Hogg CR, et al. A modified ERG technique and the results obtained in X-linked retinitis pigmentosa. Br J Ophthalmol 1983;67:419-430.

37. Nilsson J, Wright T, Westall CA. Rod a-wave analysis using high intensity flashes adds information on rod system function in 25\% of clinical ERG recordings. Vision Res 2008;48:19201925.

38. Walsh N, Valter K, Stone J. Cellular and subcellular patterns of expression of bFGF and CNTF in the normal and light stressed adult rat retina. Exp Eye Res 2001;72:495-501.

39. Xiao M, Sastry SM, Li ZY, et al. Effects of retinal laser photocoagulation on photoreceptor basic fibroblast growth factor and survival. Invest Ophthalmol Vis Sci 1998;39:618-630.

40. Li ZY, Chang JH, Milam AH. Distribution of basic fibroblast growth factor in human retinas with retinitis pigmentosa. Exp Eye Res 1997;65:855-859.

41. Penn JS, Thum LA. A comparison of the retinal effects of light damage and high illuminance light history. Prog Clin Biol Res 1987;247:425-438.

42. Rapp LM, Williams TP. A parametric study of retinal light damage in albino and pigmented rats. In: Williams TP, Baker BN (eds), The effects of constant light on visual processes. New York: Plenum Press; 1980:135-159.

43. Rapp LM, Naash MI, Wiegand RD, Joel CD, Nielsen JC, Anderson RE. Morphological and Biochemical Comparisons Between Retinal Regions Having Differing Susceptibility to Photoreceptor Degeneration. Alan R Liss Inc; 1985:421-437.

44. Tanito M, Kaidzu S, Ohira A, Anderson RE. Topography of retinal damage in light-exposed albino rats. Exp Eye Res 2008;87:292-295. 
45. Fukuda Y. A three-group classification of rat retinal ganglion cells: histological and physiological studies. Brain Res 1977;119:327-334.

46. Battelle BA, LaVail MM. Rhodopsin content and rod outer segment length in albino rat eyes: modification by dark adaptation. Exp Eye Res 1978;26:487-497.

47. Qu C, Cao W, Fan Y, Lin Y. Near-infrared light protect the photoreceptor from lightinduced damage in rats. Adv Exp Med Biol 2010;664:365-374.

48. Bush RA, Williams TP. The effect of unilateral optic nerve section on retinal light damage in rats. Exp Eye Res 1991;52:139-153.

49. Valter K, Bisti S, Gargini C, et al. Time course of neurotrophic factor upregulation and retinal protection against light-induced damage after optic nerve section. Invest Ophthalmol Vis Sci 2005;46:1748-1754.

50. Faktorovich EG, Steinberg RH, Yasumura D, Matthes MT, LaVail MM. Basic fibroblast growth factor and local injury protect photoreceptors from light damage in the rat. $J$ Neurosci 1992;12:3554-3567.

51. LaVail MM, Faktorovich EG, Hepler JM, et al. Basic fibroblast growth factor protects photoreceptors from light-induced degeneration in albino rats. Ann N Y Acad Sci 1991;638:341-347.

52. Gargini C, Bisti S, Demontis GC, Valter K, Stone J, Cervetto L. Electroretinogram changes associated with retinal upregulation of trophic factors: observations following optic nerve section. Neuroscience 2004;126:775-783.

53. Gargini C, Belfiore MS, Bisti S, Cervetto L, Valter K, Stone J. The impact of basic fibroblast growth factor on photoreceptor function and morphology. Invest Ophthalmol Vis Sci 1999;40:2088-2099.

54. Valter K, van Driel D, Bisti S, Stone J. FGFR1 expression and FGFR1-FGF-2 colocalisation in rat retina: sites of FGF-2 action on rat photoreceptors. Growth Factors 2002;20:177-188.

55. Valter K, Bisti S, Stone J. Location of CNTFRalpha on outer segments: evidence of the site of action of CNTF in rat retina. Brain Res 2003;985:169-175.

56. Bowers F, Valter K, Chan S, Walsh N, Maslim J, Stone J. Effects of oxygen and bFGF on the vulnerability of photoreceptors to light damage. Invest Ophthalmol Vis Sci 2001;42:804-815.

57. Karu T. Low-power laser therapy. Biomedical Photonics Handbook: CRC Press LLC; 2003:1-25.

58. Clarke G, Lumsden CJ, McInnes RR. Inherited neurodegenerative diseases: the one-hit model of neurodegeneration. Hum Mol Genet 2001;10:2269-2275.

59. Lampl Y, Zivin JA, Fisher M, et al. Infrared laser therapy for ischemic stroke: a new treatment strategy: results of the NeuroThera Effectiveness and Safety Trial-1 (NEST-1). Stroke 2007;38:1843-1849. 
60. Sommer AP, Pinheiro AL, Mester AR, Franke RP, Whelan HT. Biostimulatory windows in low-intensity laser activation: lasers, scanners, and NASA's light-emitting diode array system. $J$ Clin Laser Med Surg 2001;19:29-33.

61. Garbuzova-Davis S, Willing AE, Saporta S, et al. Chapter 14 Novel cell therapy approaches for brain repair. Prog Brain Res 2006;157:207-222.

62. Ilic S, Leichliter S, Streeter J, Oron A, DeTaboada L, Oron U. Effects of power densities, continous and pulse frequencies, and number of sessions of low-level laser therapy on intact rat brain. Photomedicine and Laser Surgery 2006;24:458-466.

63. Eells JT, Wong-Riley MT, VerHoeve J, et al. Mitochondrial signal transduction in accelerated wound and retinal healing by near-infrared light therapy. Mitochondrion 2004;4:559567.

64. Liang HL, Whelan HT, Eells JT, et al. Photobiomodulation partially rescues visual cortical neurons from cyanide-induced apoptosis. Neuroscience 2006;139:639-649.

65. Oron U, Yaakobi T, Oron A, et al. Attenuation of infarct size in rats and dogs after myocardial infarction by low-energy laser irradiation. Lasers Surg Med 2001;28:204-211.

66. Oron U, Yaakobi T, Oron A, et al. Low-energy laser irradiation reduces formation of scar tissue after myocardial infarction in rats and dogs. Circulation 2001;103:296-301.

67. Whelan HT, Buchmann EV, Dhokalia A, et al. Effect of NASA light-emitting diode irradiation on molecular changes for wound healing in diabetic mice. J Clin Laser Med Surg 2003;21:67-74.

68. Karu TI, Pyatibrat LV, Kalendo GS. Photobiological modulation of cell attachment via cytochrome c oxidase. Photochem Photobiol Sci 2004;3:211-216.

69. Ying R, Liang HL, Whelan HT, Eells JT, Wong-Riley MT. Pretreatment with near-infrared light via light-emitting diode provides added benefit against rotenone- and MPP+-induced neurotoxicity. Brain Res 2008;1243:167-173.

70. Natoli R, Zhu Y, Valter K, Bisti S, Eells J, Stone J. Gene and noncoding RNA regulation underlying photoreceptor protection: microarray study of dietary antioxidant saffron and photobiomodulation in rat retina. Mol Vis 2010;16:1801-1822.

71. Rutar M, Natoli R, Valter K, Provis JM. Early focal expression of the chemokine Ccl2 by Muller cells during exposure to damage-inducing bright continuous light. Invest Ophthalmol Vis Sci 2011.

72. Posten W, Wrone DA, Dover JS, Arndt KA, Silapunt S, Alam M. Low-level laser therapy for wound healing: mechanism and efficacy. Dermatol Surg 2005;31:334-340. 


\section{FIGURE LEGENDS}

Figure 1.

(A) Representative images of Toluidine Blue labelled sections from the superior region of the retina. In control retina, the cells are distinctly arranged in respective layers and outer segments are neatly organised. In LD and Sham NIR LD sections, severe disruption of the outer retina and diminished photoreceptor population are observed. No gross structural changes are seen in any of the NIR-treated groups. White line indicates how ONL thickness was measured. (B) Higher magnification images of retinal sections labelled with Toluidine blue, focussing on the outer segments, the adjacent pigment epithelium and the choroid. Thin white line highlights the OLM, thick white vertical line indicates how OS length was measured. The white horizontal trace indicates the intact outer limiting membrane. (C) Graph summarising the quantitative analysis of photoreceptor population, assessed by measuring the thickness of the ONL and the full retina (n=8). (D) Graph summarising quantitative analysis of OS lengths in the different groups $(n=8)$. To counter for variations in cutting angles, the ratio of the two measures were depicted in this graph. The black solid lines in $C$ and $D$ correspond to the area of the retina with statistical significance. ** Statistically significant differences $(P<0.05)$ compared to control groups. Scale bar represents $25 \mu \mathrm{m}$.

\section{Figure 2.}

(A) Representative dark-adapted scotopic ERG recordings showing traces of a-wave responses. Dotted lines represent ERG recording prior to treatment and/or light exposure, solid lines represent ERG recording after light exposure and NIR treatment of the same animals. Photoreceptor function was significantly reduced in non-treated LD retina (grey 
solid lines) following exposure to BL. Functional recovery was observed in Precon and Midcon but not in Postcon group (black solid lines). (B) Analysis of the relative preservation of the a-wave response of NIR-treated groups against the non-treated, LD retina. Functional changes were measured by taking the ratio of post damage plus treatment and the baseline values of a-wave amplitude in all animals in each group $(n=8)$.

(C) Analysis of the relative b-wave amplitudes of dark-adapted ERG responses in treated and non-treated SD rat retinas prior to (baseline recording) and after 7 days after exposure to bright light. Changes in b-wave amplitudes were analysed by taking the ratio of post damage plus treatment and the values from the baseline recordings in all animals in each group $(\mathrm{n}=8)$. The bars indicate the mean \pm SEM. ${ }^{* *}$ Statistically significant differences $(P<$ 0.05) compared to Control group.

\section{Figure 3.}

(A, B) Validation of the differential expression of Fgf-2 and Gfap genes using quantitative RT-PCR. cDNAs from NIR-treated and non-treated BL-exposed SD retinas were used for quantitative expression analysis and Gapdh was the reference standard. The level of expression of these genes in all groups was compared to the control group, and the value was generated for a fold change in expression. A fold-change of 1 indicates no change (dashed line), a fold change $>1$ shows upregulation and $<1$ indicates downregulation. The bars indicate the mean \pm SEM. ${ }^{* *}$ Statistically significant differences $(P<0.05)$ compared to LD group.

(C) Retinal stress status of the treated and non-treated animals after 7 days of exposure to damaging levels of light through double immunolabelling with the stress marker GFAP (red) and the neuroprotective protein FGF-2 (green). Treatment with NIR mitigated the up-regulation of both proteins in all three treatment groups. There was a 
gradient in the immunoreactivity for both proteins in the NIR treatment groups, with the strongest up-regulation in the Postcon group.

(D) Images of retinal sections labelled with CNTF antibody (red), showing a strong up-regulation in the inner retina (astrocytes) and in the remnants of ONL in the hotspot of LD retinas. In the penumbra, a few $\mu \mathrm{m}$ away, CNTF protein presence was more moderate and limited to the OS layer. A gradient of immunoreactivity in OS and GCL is demonstrated in the 3 NIR-treated groups. The blue labelling is bisbenzamide, a DNAspecific stain, indicating the nuclear layers of the retina. Scale bar represents $25 \mu \mathrm{m}$.

\section{Figure 4.}

(A) Representative images of retinal sections, from the superior retina, labelled with the marker for macrophages, ED-1 (red). White arrows point out ED-1+ cells in the NIRtreated retinas. (B) Graph summarising the quantification of macrophages present in the choroidal or retinal vasculature and the retina. ED-1+ cells were sparsely present in the choroid of the control normal retina but absent in the ONL and inner retina. One week following exposure to LD, a significant increase in the number of ED-1+ cells was observed in the choroidal and inner retinal vasculatures including an apparent tissue invasion. Following NIR treatment, the increase in the number of ED-1+ cells was more muted and localised only to the choroid and retinal vasculatures in all treated groups as indicated by the white arrows. (C) Representative images of retinal sections labelled with IBA1 (green), showing the resting and activated retinal microglial cells. In control retina, the resting microglia, identified by the ramified morphology (inset first panel in C) were present along the inner retinal vasculature only. After $7 d$ of exposure to $B C L$, there was an increase in the number of microglia that showed amoeboid morphology (inset, second panel is $C$ ) in the ONL and retinal vasculatures suggestive of its activated form. Following 
NIR treatment, activated cells were still present, but they were limited to the inner retinal vessels of all treated paradigms and not in the tissue. (D) Graph showing the number of microglial cells present, distinguishing between resting and activated cells. ${ }^{* *}$ Statistically significant differences $(P<0.05)$ compared to control groups. Scale bar represents $25 \mu \mathrm{m}$.

\section{Figure 5.}

(A) Relative a-wave (top panel) and b-wave (bottom panel) amplitudes analyses before and after 1 month of NIR treatment and BCL exposure. (B) Representative images of retinal sections stained with Toluidine Blue at 1 month recovery. The white vertical line indicates the thickness of the ONL and the black slanted line represents the outer segments length was measured. (C-D) Quantitative analyses of the OS length and ONL thickness sampled across the retina from the inferior to the superior edge $(n=8)$. The bars indicate the mean \pm SEM. ${ }^{* *}$ Statistically significant differences $(P<0.05)$ compared to control groups. Scale bar represents $25 \mu \mathrm{m}$. 
Figure 1

(A)
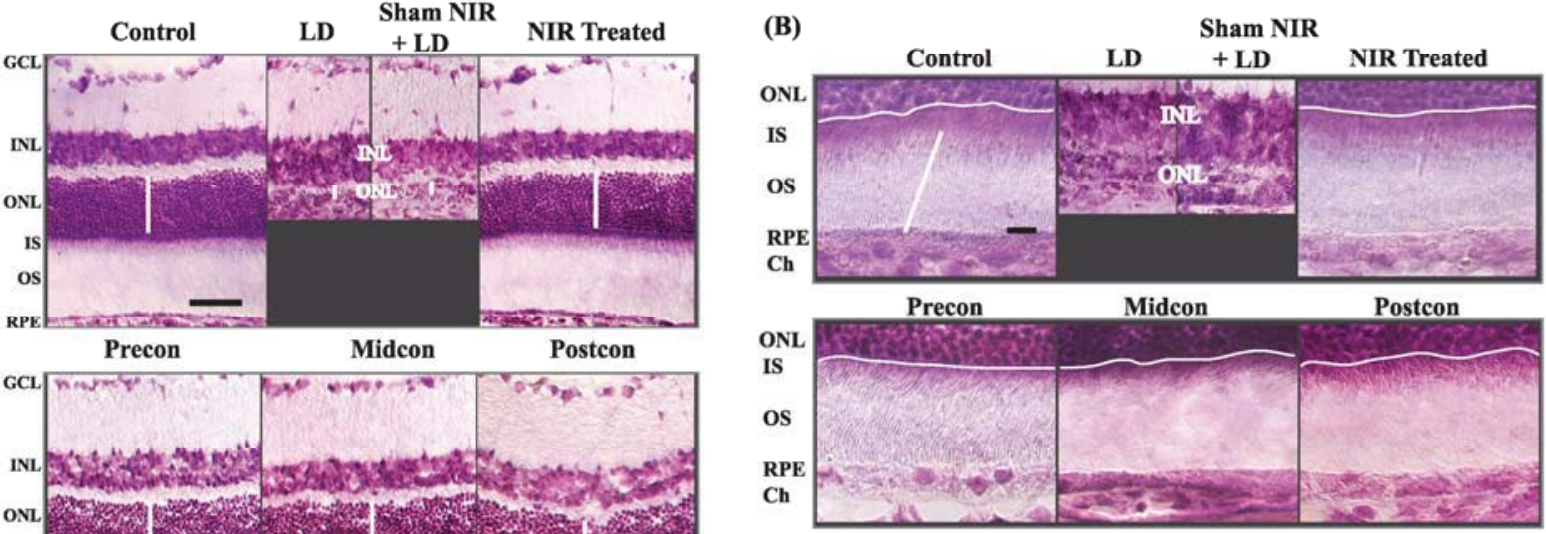

(C)
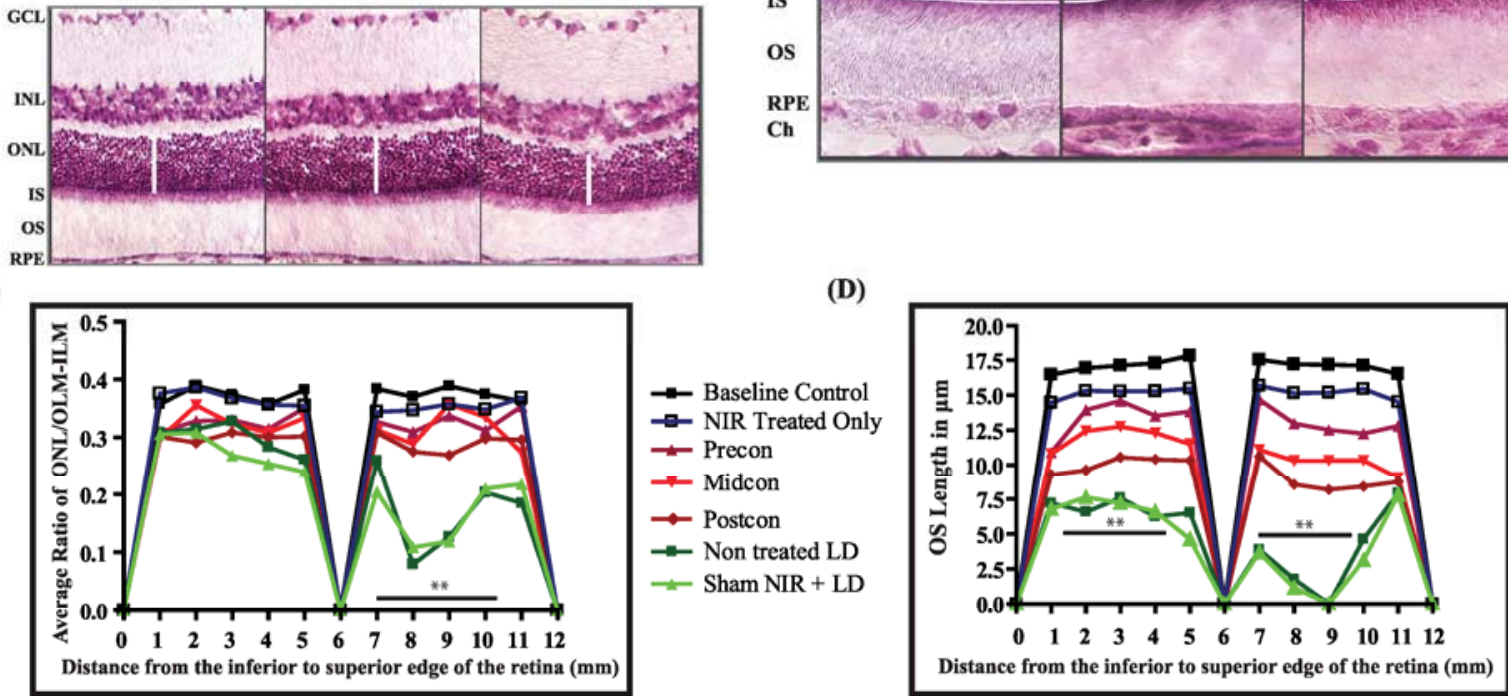
Figure 2

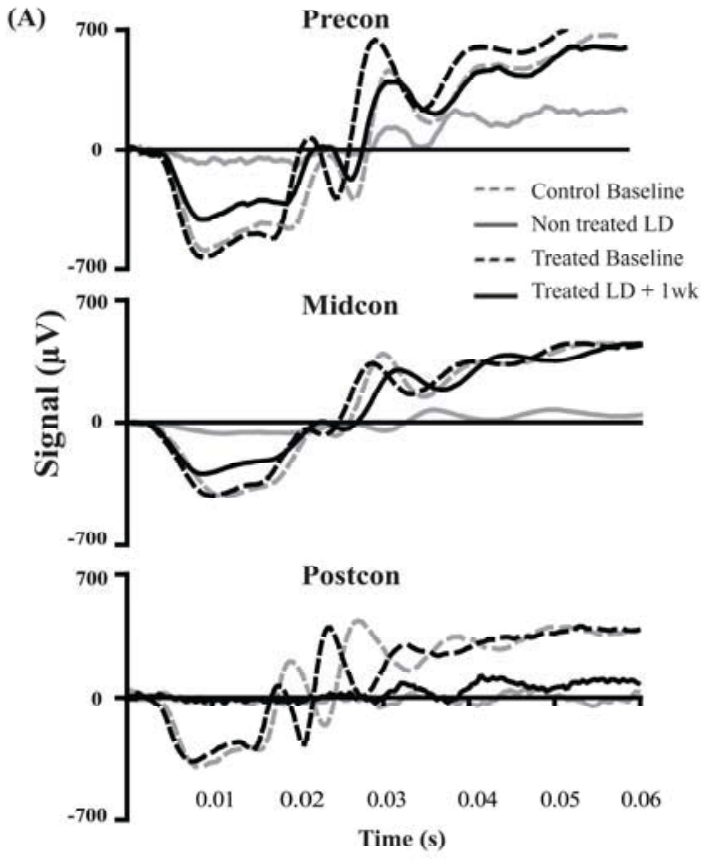

(B)

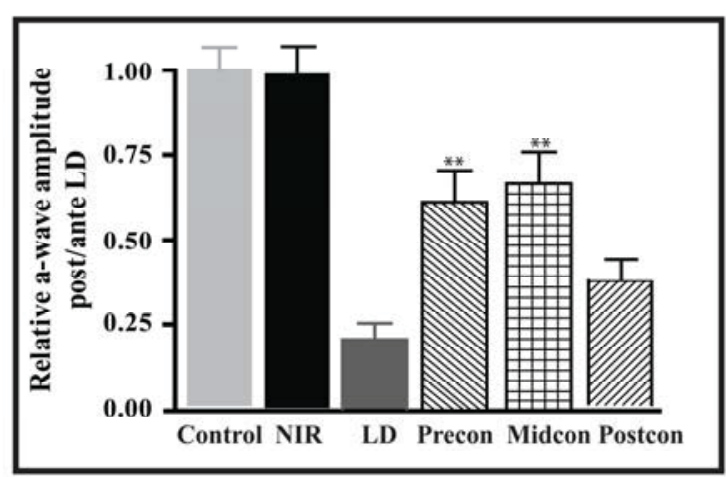

(C)

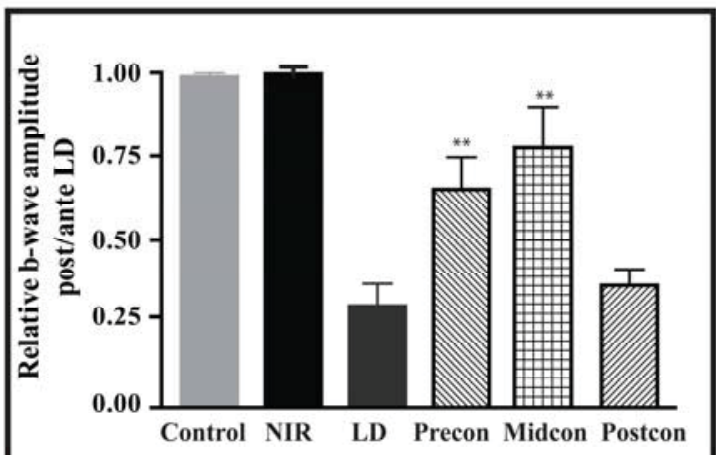


Figure 3

(A)

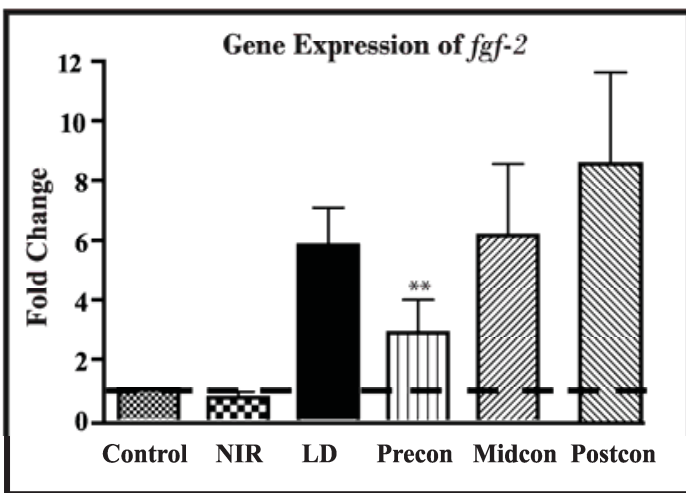

(C)

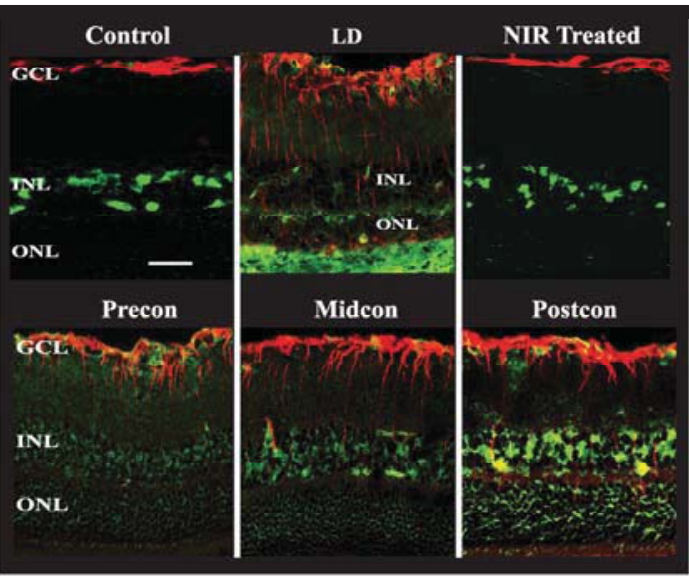

(B)

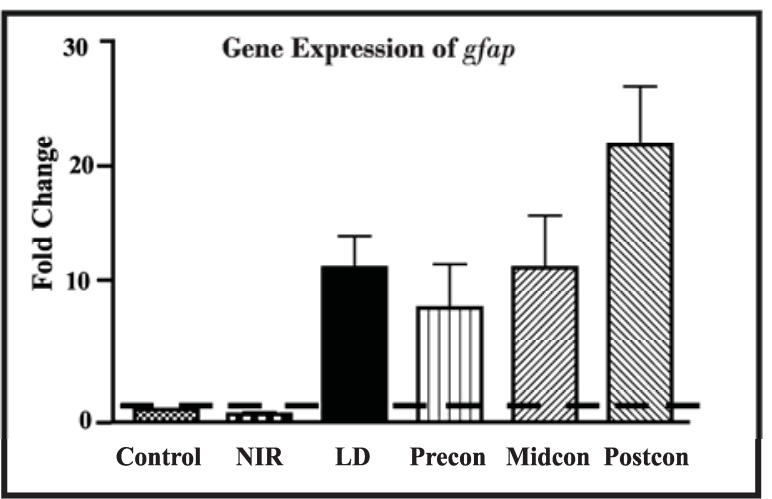

(D)

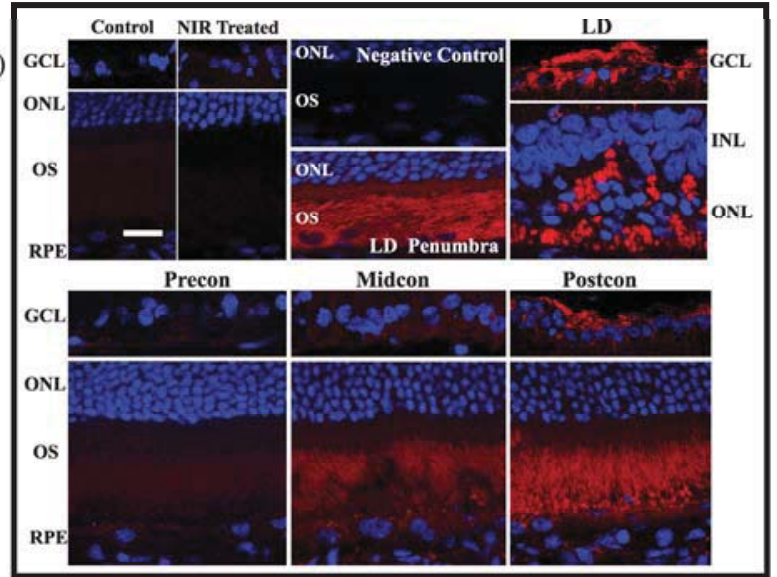


Figure 4
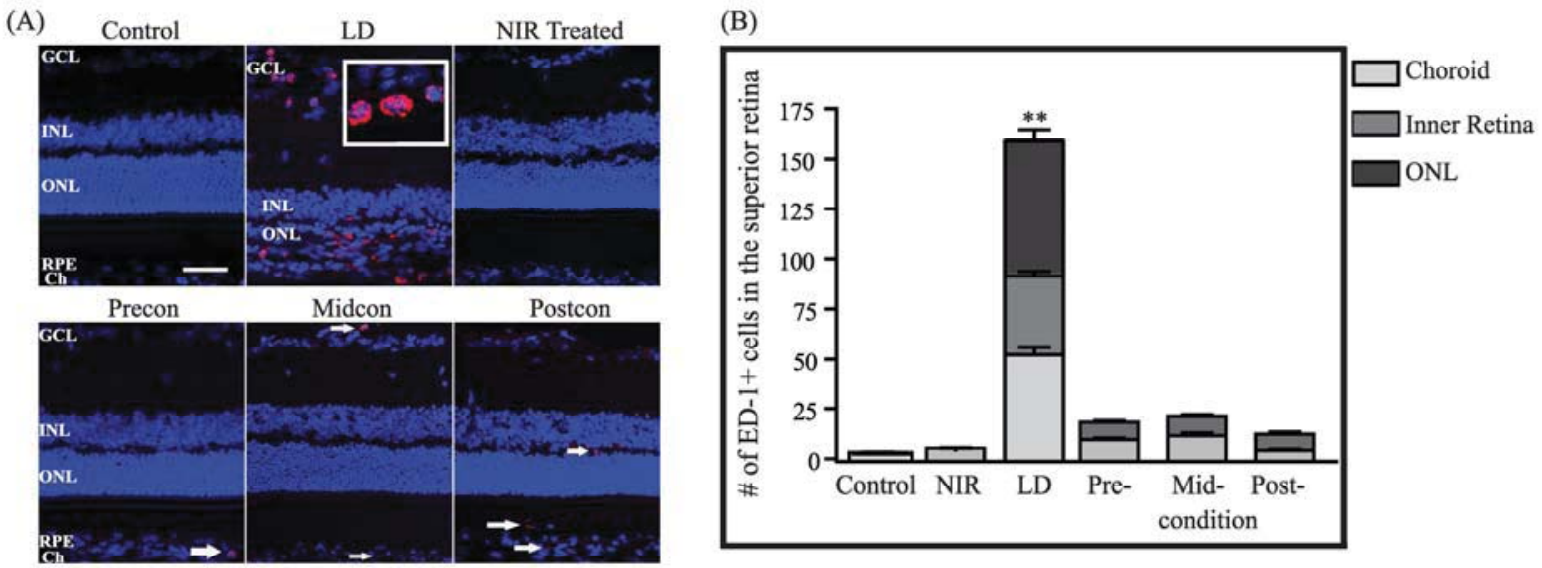

(C)

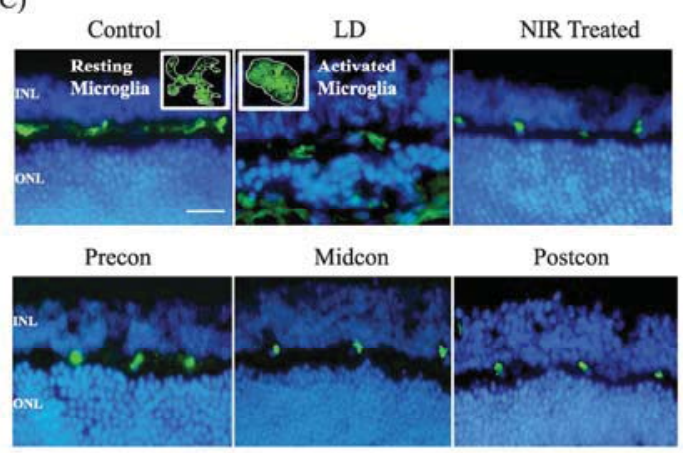

(D)

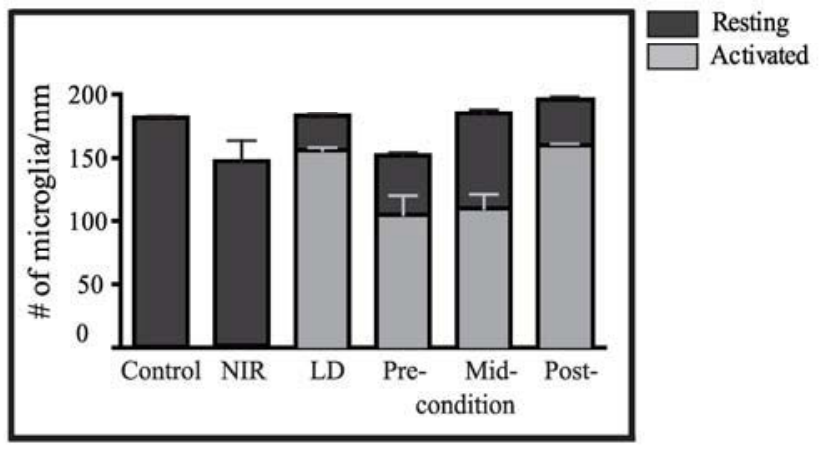


Figure 5

(A)
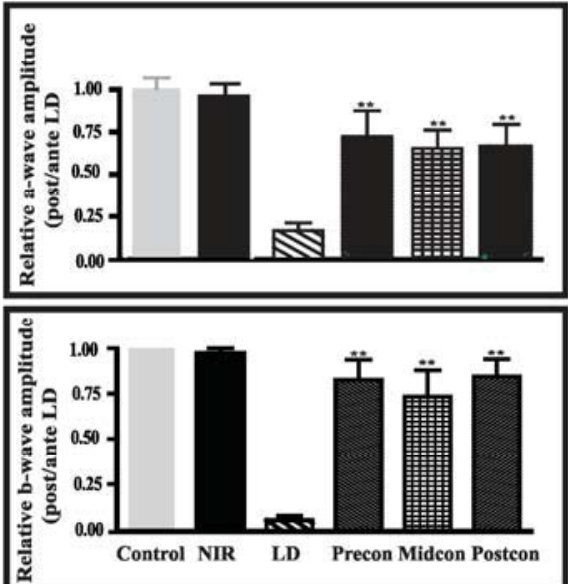

(C)

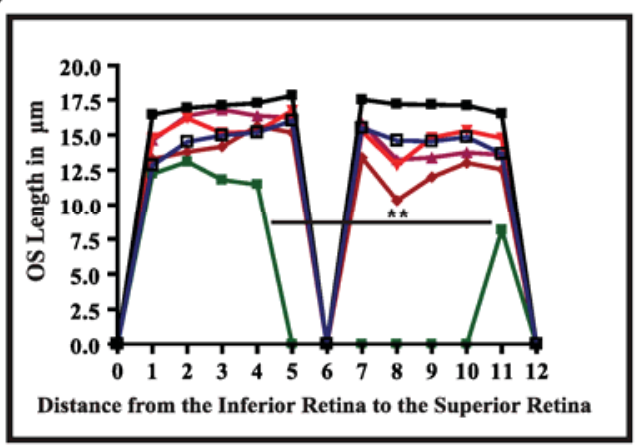

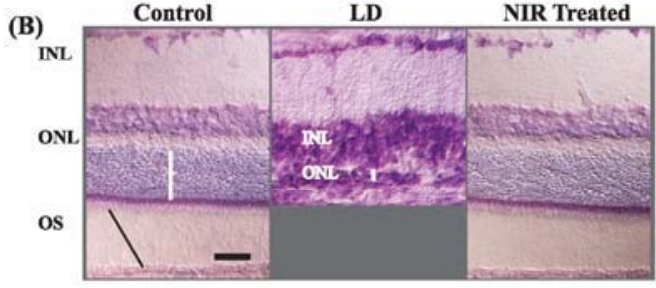

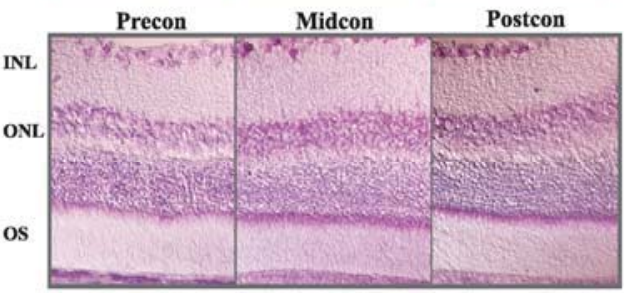

(D)

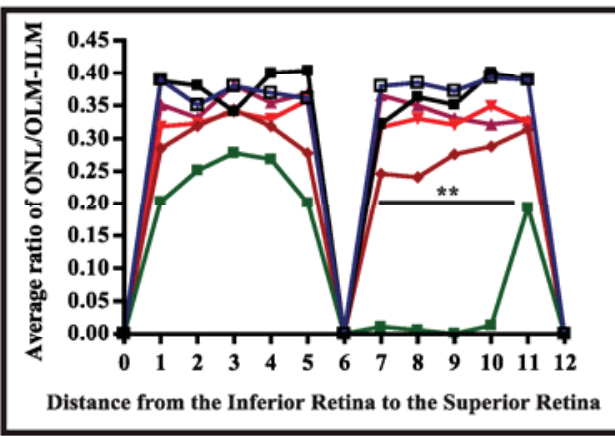

